\title{
Nonmonotonic temperature dependence of heat capacity through the glass transition within a kinetic model
}

\author{
Dwaipayan Chakrabarti and Biman Bagchi ${ }^{\mathrm{a})}$ \\ Solid State and Structural Chemistry Unit, Indian Institute of Science, Bangalore 560012, India
}

\begin{abstract}
The heat capacity of a supercooled liquid subjected to a temperature cycle through its glass transition is studied within a kinetic model. In this model, the $\beta$ process is assumed to be thermally activated and described by a two-level system. The $\alpha$ process is described as a $\beta$ relaxation mediated cooperative transition in a double well. The overshoot of the heat capacity during the heating scan is well reproduced and is shown to be directly related to delayed energy relaxation in the double well. In addition, the calculated scan rate dependencies of the glass transition temperature $T_{g}$ and the limiting fictive temperature $T_{f}^{L}$ show qualitative agreement with the known results. Heterogeneity is found to significantly reduce the overshoot of heat capacity. Furthermore, the frequency dependent heat capacity has been calculated within the present framework and found to be rather similar to the experimentally observed behavior of supercooled liquids.
\end{abstract}

\section{INTRODUCTION}

A liquid on passage through its supercooled regime to the glass displays a spectrum of thermodynamic and kinetic anomalies. ${ }^{1-4}$ Of them, the sharp rise in the measured heat capacity $^{5}$ during the heating scan of a temperature cycle through the glass transition has remained an interesting problem to study. The overshoot is often taken as a signature of a glass to liquid transition. ${ }^{6}$ The main objective of the present work is to reproduce the overshoot of the heat capacity within a kinetic model of glassy dynamics.

Time domain calorimetric experiments through the glass transformation range routinely encounter with nonequilibrium state of the sample. It is a common practice to characterize this nonequilibrium state by the fictive temperature $T_{f}$. As defined by Tool and Eichlin, ${ }^{7} T_{f}$ is the temperature at which the nonequilibrium value of a macroscopic property (e.g., enthalpy) would equal the equilibrium one. If cooling is continued through the supercooled regime, the structural relaxation eventually becomes too slow to be detected on the experimental time scale, resulting in a limiting fictive temperature $T_{f}^{L}$. Understandably, $T_{f}^{L}$ shows a dependence on the cooling rate as a slower cooling rate provides a liquid with a longer time for configurational sampling at each temperature. The calorimetric glass transition temperature $T_{g}$ is, however, found to depend on both the cooling rate $-q_{c}$ and the heating rate $q_{h}$. A shift to higher values is observed for faster rates. ${ }^{5}$ As shown elegantly by Moynihan et al., ${ }^{5}$ if the rates of cooling and heating are taken to be the same, that is $-q_{c}=q_{h}=q$, the dependence of $T_{g}$ on $q$, is given by

$$
\frac{d \ln q}{d\left(1 / T_{g}\right)}=-\Delta h^{* / R},
$$

where $\Delta h^{*}$ can be interpreted as the activation enthalpy for

\footnotetext{
a) Author to whom correspondence should be addressed. Electronic mail: bbagchi@sscu.iisc.ernet.in
}

the structural relaxation in operation and $R$ is the universal gas constant. As pointed out by Moynihan et al., it is important for the validity of the above relationship that the material be cooled and then reheated not only at the same rate but the cycle be extended well beyond the glass transformation range on either side. $T_{f}^{L}$ is also known to have an identical dependence on $q_{c},{ }^{8}$ which has recently been reproduced in model glassy systems. ${ }^{9}$

Specific heat spectroscopy, ${ }^{10,11}$ on the other hand, brings off frequency domain calorimetric measurements. As emphasized in Ref. 10, these measurements are performed with a very slow cooling rate so that the sample is in equilibrium at each temperature. What one looks here is the frequency dependent specific heat that is essentially a linear susceptibility describing the response of the sample to arbitrary small perturbations away from equilibrium. The imaginary part of the complex frequency dependent specific heat, as measured experimentally in the supercooled regime, shows a broad peak and differs from its dielectric analog in having contribution of all the degrees of freedom of the system. ${ }^{10}$ A number of theoretical approaches exist in the literature elucidating the measurable in specific heat spectroscopy. ${ }^{12-17}$

The glassy dynamics has often been considered to be a manifestation of an underlying thermodynamic transition. ${ }^{18-20}$ The celebrated Adam-Gibbs (AG) theory ${ }^{21}$ that invokes the concept of the cooperatively rearranging region (CRR) attempts to provide a connection between thermodynamics and kinetics. A different framework is provided by the so called landscape paradigm. ${ }^{22-30}$ The landscape picture consists of a connected network of potential energy minima, each minimum being surrounded by its own basin. This approach involves the division of the multidimensional configuration space into metabasins on the basis of a transition free-energy criterion. ${ }^{26}$ This can entail two vastly different time scales, the smaller one due to motions within the metabasins and the longer one due to exchange between the 


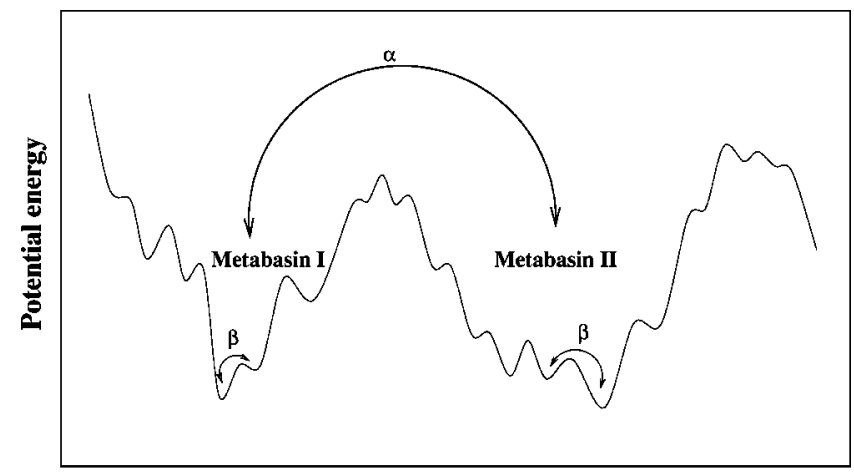

Configuration coordinates

FIG. 1. A schematic representation of the potential energy landscape showing motions within and between metabasins.

metabasins involving much larger free-energy of activation. In particular, the $\beta$ processes are visualized to originate from activated dynamics within a metabasin, while escape from one metabasin to another is taken to describe an $\alpha$ process. $^{27}$ See Fig. 1 for a schematic representation of $\beta$ and $\alpha$ processes. There have been several approaches along this line in recent times to model relaxation in supercooled liquids. ${ }^{31,32}$ It is worth noting here that the breakdown of the modecoupling theory (MCT) $)^{33-35}$ is ascribed to the dominance of relaxation by these thermally activated, large amplitude hoppings, which are unaccounted for in MCT. Recent computer simulation studies have further revealed that hopping is a highly cooperative phenomenon ${ }^{36-38}$ promoted by many body fluctuations; large amplitude hopping of a tagged particle is often preceded by somewhat larger than normal, but still small amplitude motion of several of its neighbors. Motivation of the present work largely comes from this set of findings.

In the present work, we consider a simple model of relaxation in supercooled liquids. It is a kinetic approach that attempts to combine the activated hopping in the energy landscape and the cooperative nature of the hopping event. The $\beta$ process is assumed to be a thermally activated event within a two-level system (TLS). The model assumes that an $\alpha$ process can occur only when a minimum number of $\beta$ processes are simultaneously activated. Such a treatment invoking the concept of $\beta$ organized $\alpha$ process does not seem to have been discussed previously. The present treatment, however, is consistent with an earlier view that a collection of $\beta$ excitations is a precursor of the $\alpha$ relaxation. ${ }^{39}$

The present model can be taken to belong to the class of kinetically constrained models ${ }^{40}$ that attempts to model glassy dynamics by imposing dynamical constraints on the allowed transitions between different configurations of the system, while maintaining the detailed balance. In particular, our model resembles the facilitated kinetic Ising models (FKIMs), ${ }^{41}$ originally due to Fredrickson and Andersen, in the spirit that brings in cooperativity. The key feature of their models is that a highly compressible region in the fluid can relax only if there is/are region(s) of high compressibility in the neighborhood to facilitate the relaxation. While the cooperative units (TLSs) in the present model are noninteracting, in contrast to the interacting ones in the FKIMs, the

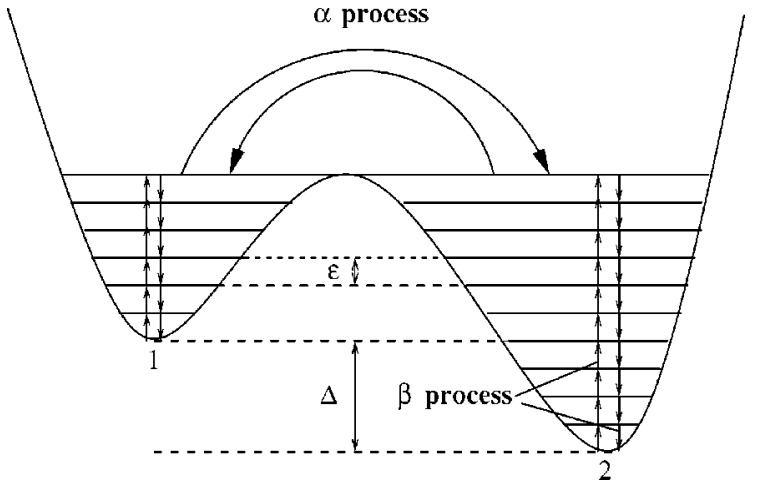

FIG. 2. A schematic representation of the model under consideration. The horizontal lines within a well represent different excitation levels. Note that the energy levels are in general degenerate, as they correspond to the sum of the energies of individual TLSs in the collection.

present model explicitly introduces the concept of $\beta$ organized $\alpha$ process within the landscape paradigm.

It is worthwhile to relate $\beta$ and $\alpha$ processes to real physical processes occurring in glass-formers. The $\alpha$ process may correspond to large-scale hopping of a particle. However, for this hopping to occur many small reorientations/ rearrangements/displacements are needed among its neighbors. The activated dynamics within a TLS may well represent small rotations. ${ }^{13}$ In the case of polymer melts which exhibit glassy behavior, the $\beta$ relaxation may be the motion of side chains. This picture apparently differs from the one drawn by Dyre while discussing solidity of viscous liquids. ${ }^{42}$ Dyre has argued that large-angle rotations are "causes" and small-angle rotations are "effects." The present picture contains the Dyre's one in the sense that small-angle rotations indeed occur following a large-scale jump motion for the completion of relaxation as evident in Fig. 2. The present model is built on a rather symmetrical picture that also necessitates small-angle rotations for a large-angle rotation to occur.

Analysis of the heat capacity during a cooling-heating cycle that extends well beyond the glass transformation range on either side shows that the present model can reproduce the overshoot of the heat capacity in the heating scan. In addition, the scan rate dependence of the glass transition temperature $T_{g}$ and that of the limiting fictive temperature $T_{f}^{L}$ are in qualitative agreement with the known results. However, a somewhat larger fall in the heat capacity prior to the overshoot than what is observed experimentally in most cases is notable. This we ascribe either to the lack of spatial heterogeneity or to the neglect of memory effects in the present treatment. The model also captures the basic features of frequency dependence of heat capacity.

The paper is organized as follows: Section II contains a detailed description of the model. Section III provides the theoretical treatment which is followed by the details of calculation in Sec. IV. The results are presented with discussion in Sec. V. Section VI concludes with a summary of the results and a critical view on the model. 


\section{MODEL}

As mentioned, we model a $\beta$ process as an activated event in a two-level system (TLS). We label the ground level of a TLS as 0 and the excited level as 1 . The waiting time before a transition can occur from the level $i(=0,1)$ is assumed to be random. The waiting time is given by the Poissonian probability density function:

$$
\psi_{i}(t)=\frac{1}{\tau_{i}} \exp \left(-t / \tau_{i}\right), \quad i=0,1,
$$

where $\tau_{i}$ is the average time of stay at the level $i$. If $p_{i}(T)$ denotes the canonical equilibrium probability of the level $i$ of a TLS being occupied at temperature $T$, detailed balance gives the following relation

$$
K(T)=\frac{p_{1}(T)}{p_{0}(T)}=\frac{\tau_{1}(T)}{\tau_{0}(T)}=\exp \left[-\epsilon /\left(k_{B} T\right)\right],
$$

where $K(T)$ is the equilibrium constant at $T$ for the two levels which have an energy separation $\epsilon$, and $k_{B}$ is the Boltzmann constant. The level 0 is taken to have a zero energy.

Within the framework of this model, a metabasin is characterized by an $N_{\beta}$ number of such non-interacting two-level systems (TLSs). A given minimum number among the total number $N_{\beta}$ of TLSs must simultaneously be in the excited levels for the occurrence of an $\alpha$ process, which then happens with a finite rate $k$. With this definition of $\alpha$ and $\beta$ processes, the heat capacity is sensitive to both the processes. A consideration of two adjacent metabasins can entail the same within the present framework. We, therefore, concentrate here on two adjacent metabasins, which we label as 1 and 2 and together call it a double well. Figure 2 shows a schematic diagram of two adjacent metabasins with illustration of dynamics within and between them. The respective numbers of TLSs that comprise the metabasins are $N_{\beta}^{(1)}$ and $N_{\beta}^{(2)}$. For a collection of $N_{\beta}^{(i)}(i=1,2)$ TLSs, a variable $\zeta_{j}^{i}(t),\left(j=1,2, \ldots, N_{\beta}^{(i)}\right)$ is defined, which takes on a value 0 if at the given instant of time $t$ the level 0 of the TLS $j$ is occupied and 1 if otherwise. $\zeta_{j}^{i}(t)$ is thus an occupation variable. The collective variables $Q_{i}(t)(i=1,2)$ are then defined as

$$
Q_{i}(t)=\sum_{j=1}^{N_{\beta}^{i}} \zeta_{j}^{i}(t)
$$

$Q_{i}(t)$ is therefore a stochastic variable in the discrete integer space $\left[0, N_{\beta}^{(i)}\right]$. Here an $\alpha$ process is assumed to occur only when all the $\beta$ processes (TLSs) in a metabasin are simultaneously excited, i.e., when $Q_{i}=N_{\beta}^{(i)}$. There is a finite rate of transition $k$ from each of the metabasins when this condition is satisfied. Within the general framework of the model, the double well becomes asymmetric when $N_{\beta}^{(1)} \neq N_{\beta}^{(2)}$, as shown in Fig. 2.

\section{THEORETICAL TREATMENT}

Theoretical analysis of nonequilibrium heat capacity is a nontrivial problem and has been addressed in great detail by Brawer, ${ }^{43,44}$ Jäckle, $^{45}$ and, in more recent time, by Odagaki and co-workers. ${ }^{46,47}$ The two widely used expressions of equilibrium heat capacity at constant volume are given by

$$
C_{v}(T)=\left(\frac{\partial E(T)}{\partial T}\right)_{v}
$$

and

$$
C_{v}(T)=\frac{\left\langle(\Delta E(T))^{2}\right\rangle}{k_{B} T^{2}}
$$

where $\left\langle(\Delta E(T))^{2}\right\rangle$ is the mean square energy fluctuation at temperature $T$. As is well known, these two are equal at equilibrium. However, they need not be equal in a nonequilibrium system.

The system, when subjected to cooling or heating at a constant rate, can be envisaged to undergo a series of instantaneous temperature changes, each in discrete step of magnitude $|\Delta T|$ in the limit $\Delta T \rightarrow 0$, at time interval of length $\Delta t$, whence $q_{i}=\Delta T / \Delta t(i=c, h) .^{5}$ A pictorial representation of the temperature control during a cooling process with finite $\Delta T$ was given by Jäckle. ${ }^{45}$ If we consider a time interval at the beginning of which the temperature has been changed from $T$ to $T^{\prime}=T+\Delta T$, the waiting time $t_{\text {obs }}$ before an observation is restricted by $\Delta t$. The heat capacity $C$, measured at a time $t_{\mathrm{obs}}$ subsequent to a temperature change from $T$ to $T^{\prime}=T+\Delta T$, is not stationary in time unless $t_{\mathrm{obs}}$ is long enough for the equilibrium to be established. The measured heat capacity, and also the energy, then become a function of the rate of cooling-heating as well, apart from $T$ and $t_{\mathrm{obs}}$. The dependence of $C$ on $q_{c}$ and/or $q_{h}$ implies that the measured heat capacity of a nonequilibrium state depends on the history of the preparation of that state. Here we restrict ourselves to the case, where $-q_{c}=q_{h}=q$. Therefore, we calculate $C\left(T, t_{\mathrm{obs}}, q\right)$ from the following equation:

$$
C\left(T, t_{\mathrm{obs}}, q\right)=\lim _{\Delta T \rightarrow 0} \frac{E\left(T+\Delta T, t_{\mathrm{obs}}, q\right)-E(T, 0, q)}{\Delta T},
$$

which is essentially a form of Eq. (5) modified to incorporate the nonequilibrium effects.

With the total energy of the system at time $t$ given by

$$
\begin{aligned}
E(T, t)= & \sum_{n=0}^{N_{\beta}^{(1)}} P_{1}(n ; T, t)\left(N_{\beta}^{(2)}-N_{\beta}^{(1)}+n\right) \quad \epsilon \\
& +\sum_{n=0}^{N_{\beta}^{(2)}} P_{2}(n ; T, t) n \quad \epsilon,
\end{aligned}
$$

where the lowest level of the well 2 is taken to have zero energy and $P_{i}(n ; T, t)$ denotes the probability that the stochastic variable $Q_{i}$ takes on a value $n$ in the $i$ th well at temperature $T$ and time $t$, the calculation of the heat capacity $C\left(T, t_{\mathrm{obs}}, q\right)$ along the cycle essentially reduces to the evaluation of $P_{i}(n ; T, t)$ 's which satisfy the following master equation: ${ }^{48}$ 


$$
\begin{aligned}
\frac{\partial P_{i}(n ; T, t)}{\partial t}= & {\left[\left(N_{\beta}^{(i)}-n+1\right) / \tau_{0}(T)\right] P_{i}(n-1 ; T, t) } \\
& +\left[(n+1) / \tau_{1}(T)\right] P_{i}(n+1 ; T, t) \\
& -\left[\left(N_{\beta}^{(i)}-n\right) / \tau_{0}(T)\right] P_{i}(n ; T, t) \\
& -\left(n / \tau_{1}(T)\right) P_{i}(n ; T, t)-k \delta_{n, N_{\beta}^{(i)}} P_{i}(n ; T, t) \\
& +k \delta_{n, N_{\beta}^{(i \pm 1)}} \delta_{j, i \pm 1} P_{j}(n ; T, t),
\end{aligned}
$$

where the "+" and "-" signs in the indices of the Kronecker delta are for $i=1$ and 2, respectively.

From a theoretical point of view, the treatment of frequency dependent heat capacity can be carried out by employing the linear response assumption. Following Nielsen and Dyre, ${ }^{17}$ the frequency dependent heat capacity $C(\omega, T)$ of our system at temperature $T$ can be given by

$$
C(\omega, T)=\frac{\left\langle E^{2}(T)\right\rangle}{k_{B} T^{2}}-\frac{s}{k_{B} T^{2}} \int_{0}^{\infty} d t e^{-s t}\langle E(T, t) E(T, 0)\rangle,
$$

where $s=i \omega, \omega$ being the frequency of the small oscillating perturbation, $i=\sqrt{-1}$, and the angular brackets denote an equilibrium ensemble averaging. The evaluation of the energy autocorrelation function can be accomplished in terms of Green's function as described in the next section.

\section{DETAILS OF CALCULATION}

We now briefly describe the details of calculation. One can have the following compact representation of the set of equations given by Eq. (9) for all possible $n$ and $i$ values

$$
\partial \mathbf{P}(T, t) \partial t=\mathbf{A}(T) \mathbf{P}(T, t),
$$

where $P_{1}(n ; T, t)$ for $n=0,1, \ldots, N_{\beta}^{(1)}$ and $P_{2}(n ; T, t)$ for $n$ $=0,1, \ldots, N_{\beta}^{(2)}$ together comprise the elements of the column vector $\mathbf{P}(T, t)$. We solve numerically by finding the eigenvalues $\{\lambda(T)\}$ and the right eigenvectors $\left\{\boldsymbol{\Phi}_{\lambda}(T)\right\}$ of the transition matrix $\mathbf{A}$ and then expanding in terms of eigenvectors $^{48}$

$$
\mathbf{P}(T, t)=\sum_{\lambda} c_{\lambda}(T) \boldsymbol{\Phi}_{\lambda}(T) \exp (\lambda(T) t) .
$$

The set of coefficients $\left\{c_{\lambda}(T)\right\}$ at each temperature $T$ is obtained from the knowledge of the initial probability distribution at $T$. In particular, $\mathbf{P}\left(T_{h}, 0\right)$ which gives the equilibrium distribution at the initial point $T_{h}$ of the temperature cycle can be obtained from the eigenvector corresponding to the zero eigenvalue of $\mathbf{A}\left(T_{h}\right)$.

For the computation of the frequency dependent heat capacity, one can write the energy autocorrelation function as

$\langle E(T, t) E(T, 0)\rangle=\sum_{i=1}^{N} \sum_{j=1}^{N} G_{T}(i, t \mid j, 0) E_{i} E_{j} P_{\mathrm{eq}}(j, T)$,

where $G_{T}(i, t \mid j, 0)$ is the Green's function that gives the probability to be in the state $i$ at a later time $t$ given that the system is in the state $j$ at time $t^{\prime}=0$, the temperature being kept constant at temperature $T, P_{\text {eq }}(j, T)$ is the equilibrium probability of the state $j$ at $T$, and $N=N_{\beta}^{(1)}+N_{\beta}^{(2)}+2$ is the

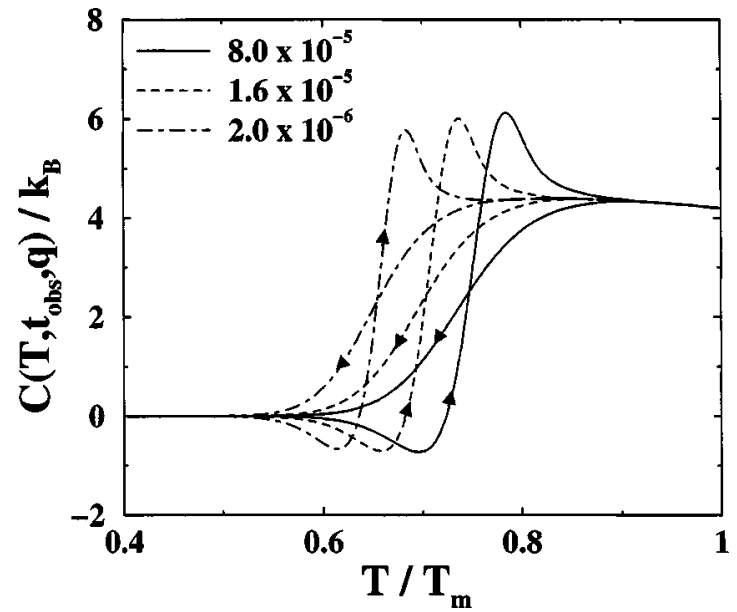

FIG. 3. The heat capacity vs reduced temperature plot for the system with $N_{\beta}^{(1)}=6$ and $N_{\beta}^{(2)}=10$, when subjected to a cooling-heating cycle with different $q$ values. The $q$ values are given in reduced units.

total number of states. Note that here the indices of states correspond to the representation followed in Eq. (11). The matrix of Green's functions satisfies the rate equation

$$
\frac{d \mathbf{G}_{T}(t)}{d t}=\mathbf{A}(T) \mathbf{G}_{T}(t),
$$

with the initial condition $\mathbf{G}_{T}(0)=\mathbf{I}$, where $\mathbf{I}$ is the identity matrix of order $N$. We write $\hat{G}_{T}(i, s \mid j)$ as the Laplace transform of $G_{T}(i, t \mid j, 0)$ :

$$
\hat{G}_{T}(i, s \mid j)=\int_{0}^{\infty} d t e^{-s t} G_{T}(i, t \mid j, 0) .
$$

The frequency dependent heat capacity is then given by

$$
\begin{aligned}
C(\omega, T)= & \frac{\left\langle E^{2}(T)\right\rangle}{k_{B} T^{2}}-\frac{s}{k_{B} T^{2}} \\
& \times \sum_{i=1}^{N} \sum_{j=1}^{N} \hat{G}_{T}(i, s \mid j) E_{i} E_{j} P_{\mathrm{eq}}(j, T),
\end{aligned}
$$

where the Green's functions can be obtained by an inversion of matrix,

$$
\hat{\mathbf{G}}_{T}(s)=(s \mathbf{I}-\mathbf{A}(T))^{-1} .
$$

\section{RESULTS AND DISCUSSIONS}

\section{A. Temperature dependence of heat capacity}

In Fig. 3, we show the heat capacity versus temperature curve obtained for our model for different cooling-heating rates. In the present calculation, we have taken $t_{\mathrm{obs}}=\Delta t$. Throughout the cycle the transition rates are assumed to be tuned with the heat bath temperature $T$. The curves look quite close to the ones observed in experiments. ${ }^{5}$ Note the sharp rise in heat capacity during heating. Figure 3 is the result of the model calculation where $N_{\beta}^{(1)}=6$ and $N_{\beta}^{(2)}$ $=10$. In the present section, temperature $T$ is expressed in reduced units of $T / T_{m}$ with $T_{m}$, the melting temperature, taken to be unity. We have set $\Delta T= \pm 0.002$ in reduced units. 


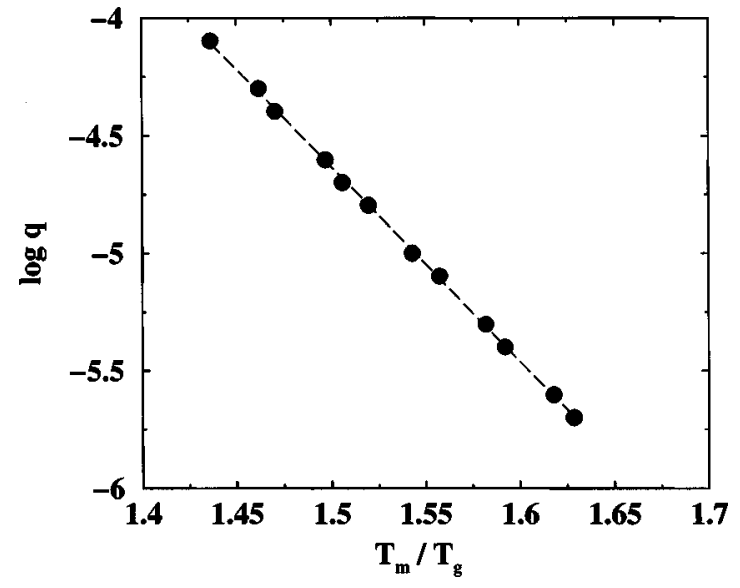

FIG. 4. The dependence of the glass transition temperature $T_{g}$ on the cooling-heating rate $q$ is shown in a plot of the logarithm of $q$ vs the reciprocal of the reduced $T_{g}$. The slope of the linear fit to the data equals -8.284 in appropriate temperature units.

The correspondence to real units is discussed later. We have also taken $\epsilon=2 k_{B} T_{m}$ and $\epsilon_{1}^{+}=18 k_{B} T_{m}$, the latter being the energy barrier to the transition from the level 1 in a TLS. The choice of such a value for $\epsilon_{1}^{*}$ ensures that the overshoot of heat capacity in the heating scan, which is often used to mark the glass transition, occurs at a temperature around $2 / 3 T_{m}$ as evident in Fig. 3. Note that the glass transition temperature $T_{g}$ is indeed found to be around two-thirds of $T_{m} \cdot{ }^{1,3}$ We express time also in reduced units, being scaled by $\tau_{1}\left(T_{m}\right)$. The cycle starts with the equilibrium population distribution at $T_{m}$. The inter-well transition rates are equal and independent of temperature. We have taken $k^{-1}=0.50$ in reduced time units. The hysteresis in the $C$ versus $T$ plot, and also the overshoot of the heat capacity observed during heating, become progressively weaker as the cooling-heating rate decreases, and eventually vanish for sufficiently slow rates. This is again in agreement with the long known experimental results.

\section{B. Scan rate dependencies of $T_{g}$ and $T_{f}^{L}$}

We have further investigated the cooling-heating rate $q$ dependence of $T_{g}$ for our model. The latter has been taken as the temperature of onset of the heat capacity increase as observed during heating. The $\log q$ versus $1 / T_{g}$ plot, as shown in the Fig. 4, is linear with a negative slope, in accordance with the experimental observations. The slope gives a measure of the energy of activation for the relaxation being in operation.

Figure 5 shows a fictive temperature $T_{f}$ versus heat bath temperature $T$ plot for different cooling rates, where $T_{f}$ is calculated in terms of energy. The freezing of structural relaxation within the experimental time scale at low temperatures is evident from the attainment of a limiting fictive temperature $T_{f}^{L}$. In the inset of Fig. 5, we show the plot of the cooling rate $q$ in the logarithmic scale versus the reciprocal of the limiting fictive temperature $T_{f}^{L}$ obtained on cooling. The linearity of the plot with a negative slope is again in good agreement with the experimental results.

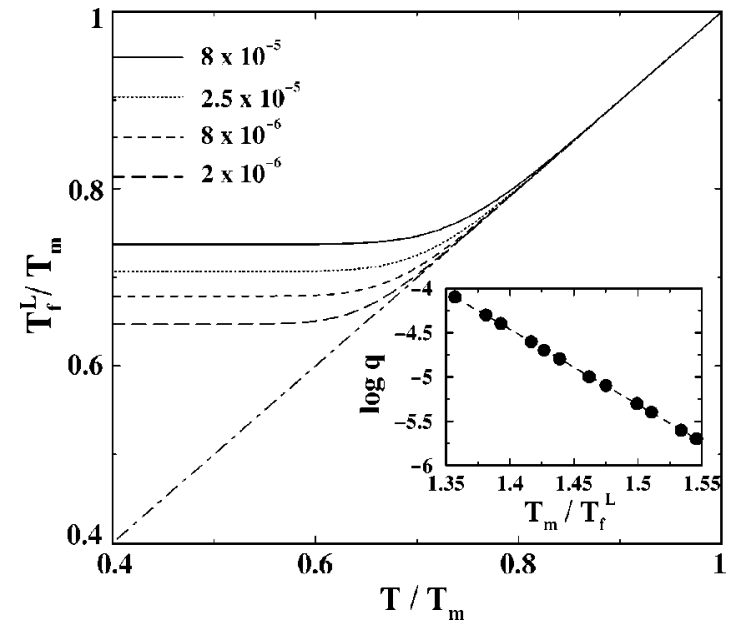

FIG. 5. Plot of the fictive temperature $T_{f}$ vs the heat bath temperature $T$ in reduced units for different cooling rates. The cooling rates are given in reduced units. The dot-dashed line traces the $T_{f}=T$ line. The inset shows the dependence of the limiting fictive temperature $T_{f}^{L}$ obtained upon cooling on the rate of cooling. The slope of the linear fit to the data is -8.488 in appropriate temperature units.

\section{Origin of the overshoot of heat capacity and the effect of heterogeneity}

The origin of the observed behavior of the calculated heat capacity can be traced back to the evolution of the energy during a cooling-heating cycle, as shown in Fig. 6. The fictive temperature evolves in an identical fashion as energy. Note that the energy or the fictive temperature remains practically unchanged during the initial period of heating before it undergoes a fall which is followed by a sharp increase. The reason is as follows. The presence of an energy barrier for all the intra-well transitions results in a slow down of the elementary relaxation rates as the system is subjected to rate cooling. The system eventually gets trapped into a nonequilibrium glassy state on continued cooling. As one subsequently starts heating, the rates of elementary relaxation keep

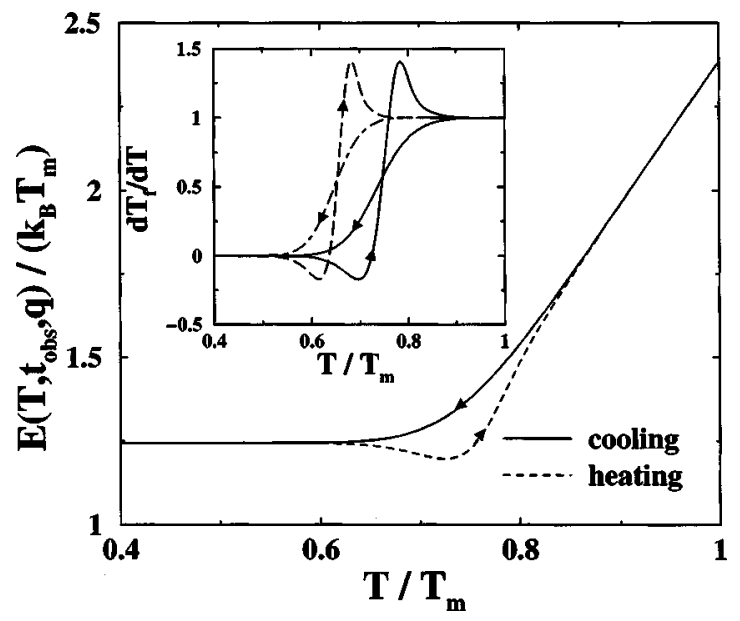

FIG. 6. The evolution of the energy of the system during a cooling-heating cycle with $N_{\beta}^{(1)}=6, N_{\beta}^{(2)}=10$, and $q=8.0 \times 10^{-5}$ in reduced units. The inset shows the $d T_{f} / d T$ vs reduced temperature plot for the system when subjected to a cooling-heating cycles. The solid line is for $q=8.0 \times 10^{-5}$, and the dot-dashed line is for $q=2.0 \times 10^{-6}$, both in reduced units. 
increasing. At first, there can be no change in the observable(s), because relaxation is still frozen within the experimental time scale. Once the heat bath temperature is high enough, what happens is a delayed (that is, overdue) energy relaxation. This explanation further gains support from the fact that the calculated heat capacity is negative. ${ }^{46}$ One should not consider this as a paradox, since the system is not in equilibrium. Such an evolution of the fictive temperature (or equivalently, the energy) during a cooling-heating cycle gives rise to the kind of $d T_{f} / d T$ versus $T$ behavior as displayed in the inset of Fig. 6 for two different coolingheating rates. This is also in accord with the experimental observation.

Note that the observed fall in heat capacity prior to the overshoot in the heating scan is somewhat larger than what is found in real experiments. Such a large apparently unphysical dip has been observed in earlier theoretical studies as well. ${ }^{49}$ This kind of sharp dip is in fact known to happen in the heating scan for cases where the relaxation process being unfrozen is exponential. ${ }^{50}$ This could be the case here also because the existence of spatially heterogeneous domains, which is believed to underlie the stretched exponential relaxation in supercooled liquids, ${ }^{51-53}$ has not been considered in the present calculations. There could also be other reasons for this limitation: First, the $\beta$ processes (two-level systems in the perspective of our model) are unlikely to be fully noninteracting. Second, the relaxation within the twolevel systems may itself be non-Markovian. That is, the delayed energy relaxation can get further delayed and can overlap with the subsequent overshoot of the heat capacity.

In the following, we explore the effect of heterogeneity. The heat capacity of the whole system can be written as a weighted average of the heat capacities of such heterogeneous domains:

$$
C=\sum_{i} w_{i} C_{i}
$$

where $C_{i}$ is the heat capacity of the domains of the $i$ th kind and $w_{i}$ is the corresponding weight. Since each of these domains relaxes with its own distinct relaxation time, the heat capacity of the system should look quite different from the one presented in Fig. 3. We illustrate this difference in Fig. 7. The heterogeneous dynamics in different domains can be included either through a distribution of $\epsilon$ (the separation between the energy levels within a TLS) or through a distribution of barrier height for transition from one level to the other within a TLS. Figure 7 shows the heat capacity behavior in a temperature cycle for different values of the barrier heights along with the average behavior. The important point here is that the domains with smaller barrier heights unfreeze and subsequently undergo the sharp rise in heat capacity earlier, which interferes (destructively) with the later drop in $C$ for domains with larger barrier heights. This could partly wipe out the comparatively large decrease in heat capacity as observed in Fig. 3. Before we conclude this sub-section, one should note that Eq. (18) holds true only when the lifetime of heterogeneity $\tau_{\mathrm{ex}}$ is much longer than $\tau_{\alpha}$, the time scale of $\alpha$ relaxation. Ediger and co-workers ${ }^{54}$ have indeed reported $\tau_{\mathrm{ex}}$ in far excess of $\tau_{\alpha}$, although Schiener et al. ${ }^{55}$ have sug-

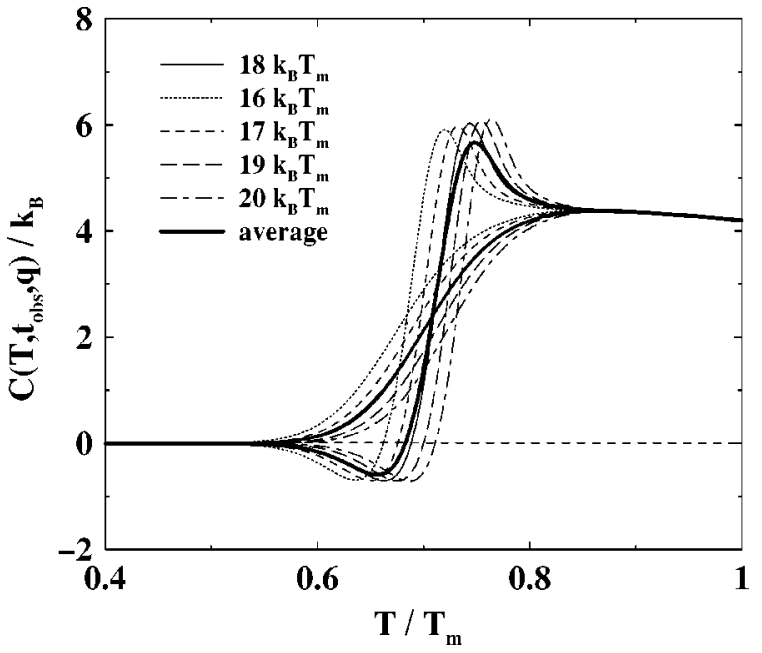

FIG. 7. The heat capacity behavior in a temperature cycle for a heterogeneous system. Here $q=2.0 \times 10^{-5}$ in reduced units. The thick solid line depicts the average behavior while the other lines as indicated in the legend correspond to different values of the barrier height $\epsilon_{1}^{\ddagger}$. The horizontal dashed line is an indicator of the zero energy. The averaging is done for illustrative purpose with arbitrary weights, the maximum weight being on the middle value and the weight gradually decreasing on both sides. The chosen values of $\epsilon_{1}^{\ddagger}$ roughly correspond to a distribution of relaxation times with a width on the order of two decades.

gested that the two time scales are comparable. Heterogeneity, however, must have a finite lifetime since exchange must occur between domains exhibiting different dynamics to maintain the ergodicity of supercooled liquids. Nevertheless, Eq. (18) is expected to provide a reasonable approximation for having at least a qualitative idea of the effect of heterogeneity, particularly the issue of the lifetime of dynamic heterogeneity being not resolved as yet. ${ }^{51}$

\section{Effect of number of TLSs in metabasins}

In Fig. 8, we show an additional feature observed for the heat capacity behavior during a temperature cycle while ex-

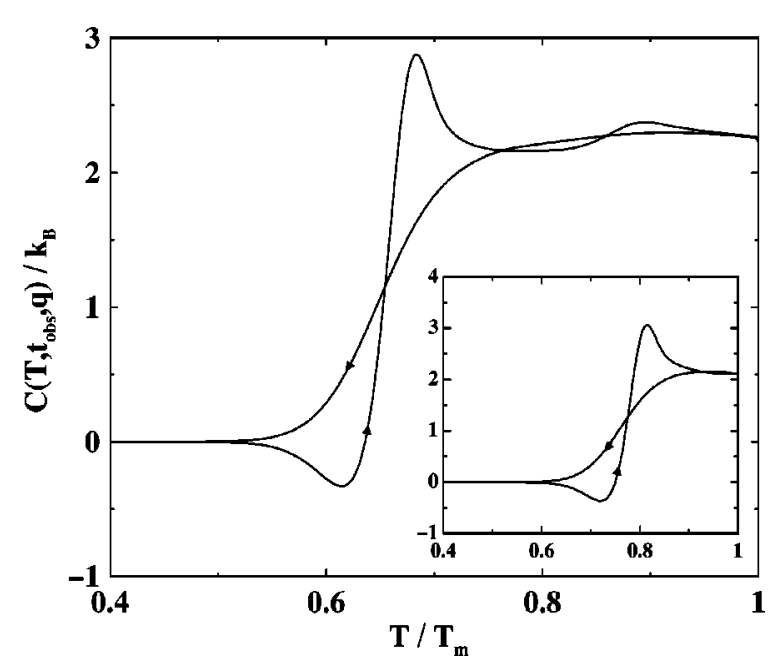

FIG. 8. The heat capacity vs reduced temperature plot for the system with $N_{\beta}^{(1)}=3$ and $N_{\beta}^{(2)}=5$, when subjected to a cooling-heating cycle with $q$ $=2 \times 10^{-6}$ in reduced units. The inset shows the same plot for $q=2$ $\times 10^{-4}$ in reduced units. The axis labels for the inset, being the same as those of the main one, are not shown. 


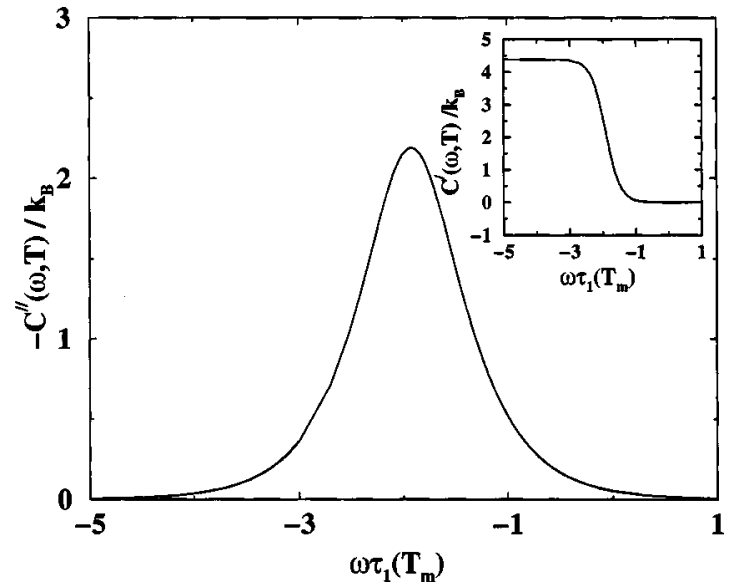

FIG. 9. Frequency dependence of the negative imaginary part of heat capacity, $-C^{\prime \prime}(\omega, T)$, for our model system with $N_{\beta}^{(1)}=6$ and $N_{\beta}^{(2)}=10$, at a given temperature $T=0.8 T_{m}$. In the inset, the real part of the frequency dependent heat capacity, $C^{\prime}(\omega, T)$, is shown for the same temperature. The frequency is scaled by the inverse of $\tau_{1}\left(T_{m}\right)$.

ploring the effect of number of TLSs in metabasins. When a different set of parameters is chosen such that the $\alpha$ relaxation becomes more probable within the observation time, a weak second peak appears at high temperatures in the heating scan. We ascribe this second peak to $\alpha$ relaxation whose effect on the temperature dependence of heat capacity gets felt for the chosen set of parameters. However, there has not been any report in the literature, to the best of our knowledge, of such an observation made experimentally. For faster rates, the second peak vanishes as evident from the inset of Fig. 8, thus substantiating the above argument.

\section{E. Frequency dependent heat capacity}

We have also investigated the frequency dependence of heat capacity as predicted by the present model. Figure 9 shows the frequency dependence of the negative imaginary part of heat capacity, $-C^{\prime \prime}(\omega, T)$, for our model system at a given temperature $T$. In the inset, the real part of the frequency dependent heat capacity $C^{\prime}(\omega, T)$ is shown for the same temperature. The spectra look similar to the ones observed experimentally for supercooled liquids. The broad peak in $-C^{\prime \prime}(\omega, T)$ is corresponding to a characteristic relaxation time and the peak frequency shifts to lower values with temperature going down as expected from the slow down of the relaxation.

\section{F. Connection with experimental systems}

The results presented in this work are all in reduced units. In order to make connection with the real world, we now present reasonable estimates of some of the parameters. For $T_{m}=300 \mathrm{~K}$, the temperature window we have looked into lies between 300 and $120 \mathrm{~K}$. Further, for $\epsilon_{1}^{\ddagger}=18 k_{B} T_{m}$ and $\tau_{a}=10^{-9} \mathrm{~s}$, the latter being the inverse of the attempt frequency, with an Arrhenius approximation to the temperature dependence of the elementary rate constants, the cooling and heating rates explored here range from 0.009 to
$0.95 \mathrm{Ks}^{-1}$. One should note that these rates are of the same order of magnitude as practised in time domain calorimetric experiments.

\section{CONCLUSION}

Let us first summarize the main features of the present work. We have presented a kinetic model that employs the concept of $\beta$ organized $\alpha$ process. In spite of the simplicity of the present model, it could reproduce many of the experimentally observed features of the anomalous behavior of heat capacity during a temperature cycle through the glass transition. The overshoot of the heat capacity during the heating scan that marks the glass transition is found to be caused by a delayed energy relaxation. The initial dip in the value of the heat capacity during the heating scan is observed to be affected by the inhomogeneity of the system. The model also captures the basic features of the frequency dependent heat capacity of supercooled liquids as observed experimentally.

The well-known bimodal frequency dependence of the dielectric relaxation in supercooled liquids can be at least qualitatively understood from the present description of $\beta$ and $\alpha$ processes. We essentially follow the description of Lauritzen and Zwanzig ${ }^{56}$ in assuming that a $\beta$ process can be taken to correspond to a two-site angular jump of individual molecules by a small angle around some axis. These individual, uncorrelated angular jumps lead to a partial relaxation of the total electric moment $\mathbf{M}(t)$ of the whole system [note that $\mathbf{M}(t)$ is the sum of the dipole moment of the individual molecules]. The dielectric susceptibility spectrum can be obtained from the auto-time correlation function of $\mathbf{M}(t)$ by using the linear response theory. ${ }^{57}$ Since $\mathbf{M}(t)$ is a sum of a relatively large number of individual dipole moments, the former is a Gaussian Markov process and thus the time correlation function of the $\beta$ relaxation mediated part must decay exponentially. As noted earlier, this $\beta$ relaxation mediated decay is incomplete because all the jumps are small and restricted. Thus, it is fair to assume the following form for the auto-time correlation function of $\mathbf{M}(t)$ :

$$
\begin{aligned}
C_{M}(t)= & \left\langle M_{\beta}^{2}\right\rangle \exp \left(-t / \tau_{\beta}\right) \\
& +\left(\left\langle M_{\circ}^{2}\right\rangle-\left\langle M_{\beta}^{2}\right\rangle\right) \exp \left(-t / \tau_{\alpha}\right),
\end{aligned}
$$

where $\tau_{\beta}$ and $\tau_{\alpha}$ are the time scales of $\beta$ and $\alpha$ relaxations, respectively. In the above equation $\left\langle M_{\beta}^{2}\right\rangle$ is the value by which the mean-square total dipole moment decays due to $\beta$ relaxation alone from the initial value of $\left\langle M_{\circ}^{2}\right\rangle$. The rest of the decay (i.e., from $\left\langle M_{\circ}^{2}\right\rangle-\left\langle M_{\beta}^{2}\right\rangle$ ) to zero occurs via the $\alpha$ process. Therefore, one can easily see the occurrence of the two time scales in the total relaxation process. However, the calculation of $\left\langle M_{\beta}^{2}\right\rangle$ would require more detailed model than the one attempted here. On the other hand, $\left\langle M_{\circ}^{2}\right\rangle$ can be obtained from the value of static dielectric constant.

While one can easily show that

$$
\tau_{\beta}=\frac{\tau_{0} \tau_{1}}{\tau_{0}+\tau_{1}},
$$

the estimation of $\tau_{\alpha}$ is much more difficult. However, a crude estimate of the latter can be obtained in the following 
way. One can consider an absorbing boundary at $Q_{i}=N_{\beta}^{(i)}$, and calculate the mean first passage time $\tau^{(i)}$ that would give a relaxation time for a cooperative transition out of the well $i{ }^{58}$ Once such a relaxation time is determined for each of the two wells, these relaxation times can be used to obtain $\tau_{\alpha}$ through an equation similar to Eq. (20). Equation (19) can then be used to compute the frequency dependent dielectric constant $\epsilon(\omega)$. Because of a wide separation of $\tau_{\alpha}$ and $\tau_{\beta}$, the imaginary part of $\epsilon(\omega)$ would exhibit the bimodal dispersion. The above qualitative analysis remains unchanged when $\mathbf{M}(t)$ corresponds to that of a heterogeneous domain and the domains are assumed to be noninteracting. The latter may not be a bad approximation for a not too strongly dipolar liquid (for example, chlorobenzene).

The present model can be considered as a representative in a simple form of a class of wider, more sophisticated and more general models. An immediate generalization will be to include the correlations among the $\beta$ processes within a metabasin. The success of our model in reproducing many aspects of experimentally observed heat capacity behavior during the temperature cycle is noteworthy. Most importantly, we need not invoke any singularity, thermodynamic or kinetic, to explain the anomalous heat capacity behavior. The present work suggests that the heat capacity anomaly has a purely kinetic origin. While the elementary relaxation rates evolve with the heat bath temperature, it is the slow population $(\beta)$ relaxation within a well that gives rise to the delayed energy relaxation. In this model, the much slower $\alpha$ relaxation is seen to get quenched when these $\beta$ processes themselves slow down. In this picture, $\beta$ relaxation is not only a precursor to $\alpha$ relaxation but the latter can occur only when a coherence between several $\beta$ processes occurs.

\section{ACKNOWLEDGMENTS}

We gratefully acknowledge Professor C. A. Angell and Dr. R. Murarka for useful discussions and/or comments on the manuscript. This work was supported in parts by grants from CSIR, DAE and DST, India. D.C. acknowledges the University Grants Commission (UGC), India for providing the Research Fellowship.

${ }^{1}$ C. A. Angell, K. L. Ngai, G. B. McKenna, P. F. McMillan, and S. W. Martin, J. Appl. Phys. 88, 3113 (2000).

${ }^{2}$ M. D. Ediger, C. A. Angell, and S. R. Nagel, J. Phys. Chem. 100, 1322 (1996).

${ }^{3}$ P. G. Debenedetti and F. H. Stillinger, Nature (London) 410, 259 (2001).

${ }^{4}$ U. Mohanty, Adv. Chem. Phys. 89, 89 (1994).

${ }^{5}$ C. T. Moynihan, A. J. Easteal, J. Wilder, and J. Tucker, J. Phys. Chem. 78, 2673 (1974).

${ }^{6}$ I. M. Hodge, J. Non-Cryst. Solids 169, 211 (1994).

${ }^{7}$ A. Tool and C. G. Eichlin, J. Am. Chem. Soc. 14, 276 (1931).

${ }^{8}$ C. T. Moynihan, A. J. Easteal, M. A. DeBolt, and J. Tucker, J. Am. Chem. Soc. 59, 12 (1976).
${ }^{9}$ B. Halpern and J. Bisquert, J. Chem. Phys. 114, 9512 (2001).

${ }^{10}$ N. O. Birge and S. R. Nagel, Phys. Rev. Lett. 54, 2674 (1985).

${ }^{11}$ T. Christensen, J. Phys. Colloq. 46, C8-635 (1985).

${ }^{12}$ J. Jäckle, Z. Phys. B: Condens. Matter 64, 41 (1986).

${ }^{13}$ D. W. Oxtoby, J. Chem. Phys. 85, 1549 (1986).

${ }^{14}$ R. Zwanzig, J. Chem. Phys. 88, 5831 (1988).

${ }^{15}$ M. Cieplak and G. Szamel, Phys. Rev. B 37, 1790 (1988).

${ }^{16}$ J. Jäckle, Physica A 162, 377 (1990).

${ }^{17}$ J. K. Nielsen and J. C. Dyre, Phys. Rev. B 54, 15754 (1996).

${ }^{18}$ J. H. Gibbs and E. A. DiMarzio, J. Chem. Phys. 28, 373 (1958).

${ }^{19}$ T. R. Kirkpatrick, D. Thirumalai, and P. G. Wolynes, Phys. Rev. A 40, 1045 (1989).

${ }^{20}$ X. Xia and P. G. Wolynes, Proc. Natl. Acad. Sci. U.S.A. 97, 2990 (2000).

${ }^{21}$ G. Adam and J. H. Gibbs, J. Chem. Phys. 43, 139 (1965).

${ }^{22}$ M. Goldstein, J. Chem. Phys. 51, 3728 (1969).

${ }^{23}$ G. P. Johari and M. Goldstein, J. Chem. Phys. 53, 2372 (1970); 55, 4245 (1971).

${ }^{24}$ F. H. Stillinger and T. A. Weber, Phys. Rev. A 25, 978 (1982).

${ }^{25}$ F. H. Stillinger and T. A. Weber, Phys. Rev. A 28, 2408 (1983).

${ }^{26}$ F. H. Stillinger, Phys. Rev. B 41, 2409 (1990).

${ }^{27}$ F. H. Stillinger, Science 267, 1935 (1995).

${ }^{28}$ U. Mohanty, I. Oppenheim, and C. H. Taubes, Science 266, 425 (1994).

${ }^{29}$ S. Sastry, P. G. Debenedetti, and F. H. Stillinger, Nature (London) 393, 554 (1998).

${ }^{30}$ S. Sastry, Nature (London) 409, 164 (2001).

${ }^{31}$ G. Diezemann, J. Chem. Phys. 107, 10112 (1997).

${ }^{32}$ G. Diezemann, U. Mohanty, and I. Oppenheim, Phys. Rev. E 59, 2067 (1999).

${ }^{33}$ U. Bengtzelius, W. Götze, and A. Sjölander, J. Phys. C 17, 5915 (1984).

${ }^{34}$ W. Götze and L. Sjögren, Rep. Prog. Phys. 55, 241 (1992).

${ }^{35}$ C. A. Angell, J. Phys. Chem. Solids 49, 863 (1988).

${ }^{36}$ G. Wahnström, Phys. Rev. A 44, 3752 (1991).

${ }^{37}$ H. Miyagawa, Y. Hiwatari, B. Bernu, and J. P. Hansen, J. Chem. Phys. 88, 3879 (1988)

${ }^{38}$ S. Bhattacharyya and B. Bagchi, Phys. Rev. Lett. 89, 025504 (2002).

${ }^{39}$ G. P. Johari, private communication (2003).

${ }^{40}$ F. Ritort and P. Sollich, Adv. Phys. 52, 219 (2003).

${ }^{41}$ G. H. Fredrickson and H. C. Andersen, Phys. Rev. Lett. 53, 1244 (1984); J. Chem. Phys. 83, 5822 (1985).

42 J. C. Dyre, Phys. Rev. E 59, 2458 (1999).

${ }^{43}$ S. A. Brawer, Relaxation in Viscous Liquids and Glasses (McGraw-Hill, New York, 1983).

${ }^{44}$ S. A. Brawer, J. Chem. Phys. 81, 954 (1984)

${ }^{45}$ J. Jäckle, Rep. Prog. Phys. 49, 171 (1986).

${ }^{46}$ T. Tao, A. Yoshimori, and T. Odagaki, Phys. Rev. E 64, 046112 (2001); 66, 041103 (2002).

${ }^{47}$ T. Odagaki, T. Yoshidome, T. Tao, and A. Yoshimori, J. Chem. Phys. 117, 10151 (2002).

${ }^{48}$ N. G. van Kampen, Stochastic Processes in Physics and Chemistry (Elsevier Science B. V., Amsterdam, 1992).

${ }^{49}$ M. H. Cohen and G. S. Grest, Adv. Chem. Phys. 48, 370 (1981).

${ }^{50} \mathrm{We}$ are indebted to C. A. Angell for pointing this out.

${ }^{51}$ M. D. Ediger, Annu. Rev. Phys. Chem. 51, 99 (2000).

${ }^{52}$ H. Sillescu, J. Non-Cryst. Solids 243, 81 (1999).

${ }^{53}$ R. Richert, J. Phys.: Condens. Matter 14, R703 (2002).

${ }^{54}$ M. T. Cicerone, F. R. Blackburn, and M. D. Ediger, J. Chem. Phys. 102, 471 (1995); M. T. Cicerone and M. D. Ediger, ibid. 103, 5684 (1995).

${ }^{55}$ B. Schiener, R. Bömer, A. Loidl, and R. V. Chemberlin, Science 274, 752 (1996).

${ }^{56}$ J. I. Lauritzen, Jr. and R. Zwanzig, Adv. Mol. Relax. Processes 5, 339 (1973).

${ }^{57}$ R. Zwanzig, Nonequilibrium Statistical Mechanics (Oxford University Press, New York, 2001)

${ }^{58}$ D. Chakrabarti and B. Bagchi, J. Chem. Phys. 118, 7965 (2003). 